\title{
Star-forming galaxies with hot dust emission in the Sloan Digital Sky Survey discovered by the Wide-field Infrared Survey Explorer (WISE)
}

\author{
Y. I. Izotov ${ }^{1,2,3}$, N. G. Guseva ${ }^{1,2}$, K. J. Fricke ${ }^{1,4}$, and C. Henkel ${ }^{1,5}$
}

\author{
Max-Planck-Institut für Radioastronomie, Auf dem Hügel 69, 53121 Bonn, Germany \\ e-mail: izotov@mao.kiev.ua \\ 2 Main Astronomical Observatory, Ukrainian National Academy of Sciences, Zabolotnoho 27, Kyiv 03680, Ukraine \\ 3 LUTH, Observatoire de Paris, CNRS, Université Paris Diderot, Place Jules Janssen 92190 Meudon, France \\ 4 Institut für Astrophysik, Göttingen Universität, Friedrich-Hund-Platz 1, 37077 Göttingen, Germany \\ 5 Astronomy Department, King Abdulaziz University, PO Box 80203, Jeddah, Saudi Arabia
}

Received 4 November 2011 / Accepted 22 November 2011

\section{ABSTRACT}

\begin{abstract}
We present the results of a search for Sloan Digital Sky Survey (SDSS) emission-line galaxies with very red 3.4 $\mu \mathrm{m}-4.6 \mu \mathrm{m}(W 1-W 2)$ colours in the Wide-field Infrared Survey Explorer (WISE) Preliminary Release Source Catalogue (PRSC) aiming to find objects with hot dust emission. For this purpose we considered a sample of $\sim 16000$ galaxies with strong emission lines selected out of a total of $\sim 900000$ SDSS spectra and identified them with the PRSC sources. We find that $\sim 5000$ sources out of the $\sim 16000$ SDSS galaxies are present in the PRSC, including $\sim 1000$ galaxies with sufficiently strong [O III] $\lambda 4363$ emission lines to permit reliable determinations of the oxygen abundance. No correlation of $W 1-W 2$ with metallicity is found. On the other hand, there is clear evidence for a redder $W 1-W 2$ index in galaxies with higher $\mathrm{H} \beta$ luminosity and higher $\mathrm{H} \beta$ equivalent width, implying that strong UV radiation from young starbursts efficiently heats interstellar dust to high temperatures. However, galaxies with very red colours $W 1-W 2>2$ mag, similar to that in the local extreme star-forming galaxy SBS 0335-052E, are very rare. In addition to three previously known sources, which are not present in our sample, we found only four such galaxies.
\end{abstract}

Key words. galaxies: fundamental parameters - galaxies: starburst - galaxies: ISM - galaxies: abundances

\section{Introduction}

Nearby star-forming emission-line galaxies play an important role for our understanding of star-formation processes in low-metallicity environments, and they can be considered as local counterparts or "analogs" of high-redshift starforming Lyman-break galaxies (LBGs). Recently, Heckman et al. (2005) identified nearby $(z<0.3)$ ultraviolet-luminous galaxies (UVLGs) selected from the Galaxy Evolution Explorer (GALEX). Eventually, compact UVLGs were called Lymanbreak analogs (LBAs). They resemble LBGs in several respects. In particular, their metallicities are subsolar, and their star-formation rates (SFRs) of $\sim 4-25 M_{\odot} \mathrm{yr}^{-1}$ are overlapping with those for LBGs. Recently, Cardamone et al. (2009) selected a sample of 251 compact strongly star-forming galaxies at $z \sim 0.112-0.36$ on the basis of their intense green colour on the Sloan Digital Sky Survey (SDSS) images ("green pea" galaxies), which again are similar to LBGs owing to their low metallicity and high SFRs. Izotov et al. (2011) extracted a sample of 803 star-forming luminous compact galaxies (LCGs) with hydrogen $\mathrm{H} \beta$ luminosities $L(\mathrm{H} \beta) \geq 3 \times 10^{40} \mathrm{erg} \mathrm{s}^{-1}$ and $\mathrm{H} \beta$ equivalent widths $\mathrm{EW}(\mathrm{H} \beta) \geq 50 \AA$ from SDSS spectroscopic data. These galaxies have properties similar to "green pea" galaxies but are distributed over a wider range of redshifts $z \sim 0.02-0.63$. The SFRs of LCGs are high $\sim 0.7-60 M_{\odot} \mathrm{yr}^{-1}$ and overlap with those of LBGs. Izotov et al. (2011, see also Guseva et al. 2009) showed that LBGs, LCGs, luminous metal-poor star-forming galaxies (Hoyos et al. 2005), extremely metal-poor emission-line galaxies at $z<1$ (Kakazu et al. 2007), and low-redshift blue compact dwarf (BCD) galaxies with strong star-formation activity obey a common luminosity-metallicity relation. Therefore, it is important to study nearby star-forming galaxies over a wide range of luminosities and metallicities to shed light on physical conditions and star-formation history in high-redshift galaxies even though most metal-deficient and low-luminosity highredshift galaxies are still awaiting their detection.

The Infrared Space Observatory (ISO), Spitzer, and most recently the Wide-field Infrared Survey Explorer (WISE, Wright et al. 2010) open up the opportunity to probe properties of star-forming galaxies in the mid-infrared range (MIR) 3.5$24 \mu \mathrm{m}$, the range of warm and hot dust. The WISE mission has an advantage because it is directed to produce an all-sky photometric survey at wavelengths $3.4 \mu \mathrm{m}(W 1), 4.6 \mu \mathrm{m}(W 2), 12 \mu \mathrm{m}$ (W3) and $22 \mu \mathrm{m}(W 4)$ with a sensitivity at $\sim 12-24 \mu \mathrm{m}$ that is $~ 1000$ times higher than that of the InfraRed Astronomical Satellite (IRAS) and has an angular resolution of $\sim 6^{\prime \prime}$ at $3.4 \mu \mathrm{m}$.

Thuan et al. (1999) first showed (from ISO spectroscopy) that one of the most metal-deficient BCDs, SBS 0335-052E (e.g. Izotov et al. 1990), is extraordinarily bright in the MIR range, implying a large amount of warm $(\sim 100-300 \mathrm{~K})$ dust. Later, Houck et al. (2004) based on Spitzer spectra have confirmed the presence of warm dust in SBS 0335-052E and found that the dust emission peaks at a wavelength of $\sim 28 \mu \mathrm{m}$, much shorter than that for the bulk of star-forming galaxies. Ground-based infrared spectroscopy of SBS 0335-052E by Hunt et al. (2001) 

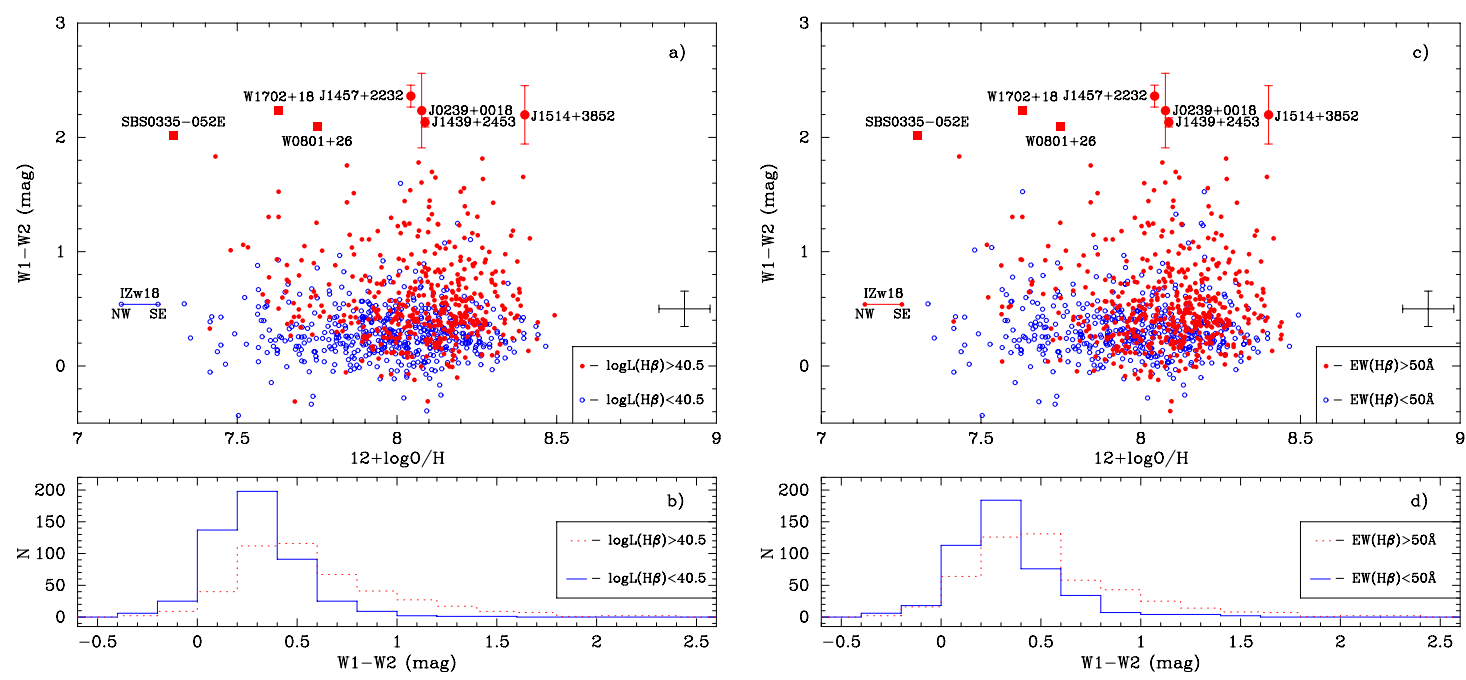

Fig. 1. a) Dependence of the $W 1-W 2$ colour on the oxygen abundance $12+\log \mathrm{O} / \mathrm{H}$ for a sample of $\sim 1000$ galaxies. Galaxies with $\mathrm{H} \beta$ luminosity $L(\mathrm{H} \beta) \geq 3 \times 10^{40} \mathrm{erg} \mathrm{s}^{-1}$, corresponding to $\mathrm{SFR}(\mathrm{H} \beta) \geq 0.7 M_{\odot} \mathrm{yr}^{-1}$, are shown by red filled circles while galaxies with $L(\mathrm{H} \beta)<3 \times 10^{40} \mathrm{erg} \mathrm{s}{ }^{-1}$ are shown by blue open circles. The NW and SE components of the low-metallicity BCD I Zw 18 are labelled and connected with a solid line. Newly identified galaxies with $W 1-W 2>2$ mag are shown by large red filled circles, the three galaxies discussed by Griffith et al. (2011) are shown by large red filled squares. Typical error bars are shown in the lower right corner. b) Histograms of $W 1-W 2$ distributions for galaxies with $L(\mathrm{H} \beta) \geq$ $3 \times 10^{40} \mathrm{erg} \mathrm{s}^{-1}$ (red dotted line) and with $L(\mathrm{H} \beta)<3 \times 10^{40} \mathrm{erg} \mathrm{s}^{-1}$ (blue solid line). c) and d) same as a) and b), respectively, but the sample is split into galaxies with $E W(\mathrm{H} \beta) \geq 50 \AA$ (red symbols and lines) and $E W(\mathrm{H} \beta)<50 \AA$ (blue symbols and lines).

revealed that the continuum at shorter wavelengths in the range $3.4-4 \mu \mathrm{m}$ is rising in the direction of longer wavelengths. Later, Griffith et al. (2011) found that the WISE 3.4-4.6 $\mu \mathrm{m}(W 1-W 2)$ colour of SBS 0335-052E is very red, >2 mag. This longward rising of MIR emission implies the presence of hot (up to $1000 \mathrm{~K}$ ) dust emission.

The properties of SBS 0335-052E in the MIR range are very different from that in another extremely metal-deficient BCD with similar metallicity, I Zw 18. Hirashita \& Hunt (2004) attributed these differences to different modes of star formation, an "active" mode in very compact regions of SBS 0335-052E and a "passive" mode in the more diffuse interstellar medium of I Zw 18.

Griffith et al. (2011) argued that WISE can be an efficient tool in searching for other star-forming galaxies with appreciable hot dust emission and demonstrated the truth of this statement by finding of three low-metallicity BCDs with $W 1-W 2>2$ mag. However, they noted that these galaxies are rare. In the present paper we attempt to find new star-forming galaxies selected from the Data Release 7 (DR7) of the SDSS with red WISE $W 1-W 2$ colours in the Preliminary Release Source Catalogue (PRSC), which covers $57 \%$ of the sky.

\section{Selection criteria}

We used the whole spectroscopic data base of the SDSS DR7 (Abazajian et al. 2009), which comprises 2900000 spectra of galaxies to select a sample of $\sim 16000$ spectra with strong emission lines, excluding those that show evidence for AGN activity. Thus, our sample includes the majority of SDSS star-forming galaxy spectra with $E W(\mathrm{H} \beta) \geq 10 \AA$. A small fraction of these spectra $(\sim 2 \%)$ represents individual $\mathrm{H}$ II regions in nearby spirals but the overwhelming majority is composed of integrated spectra of galaxies from farther distances. The coordinates of SDSS selected galaxies are used to identify sources in the WISE PRSC within a circular aperture of $10^{\prime \prime}$ in radius. In total, 5000 WISE sources were identified with SDSS galaxies out of the sample of $\sim 16000$ galaxies. Nearly 1000 galaxies out of $\sim 5000$ galaxies detected by WISE have sufficiently strong [O III] $\lambda 4363$ emission lines in their SDSS spectra with a line flux error not exceeding $50 \%$, allowing for a reliable oxygen abundance determination. We analyse the MIR properties of this sample of $\sim 1000$ galaxies in Sect. 3.

\section{Results}

Several galaxy components can contribute to the emission in the 3.4-4.6 $\mu \mathrm{m}$ range: stars, ionised gas, polycyclic aromatic hydrocarbon (PAH) emission, and hot dust. Generally, the PAH emission is weak in a low-metallicity environment (Engelbracht et al. 2008; Hunt et al. 2010). Stellar (with an effective temperature $\geq 3000 \mathrm{~K}$ ) and interstellar ionised gas emission is characterised by $W 1-W 2$ colours of $\sim 0-0.4$ mag. In particular, the $W 1-W 2 \sim$ 0.5 mag of I Zw 18, one of the most metal-deficient BCDs, is consistent with that of stellar and ionised gas emission only. On the other hand, a colour excess above $W 1-W 2 \sim 0.4$ mag could be an indication of hot dust with a temperature of several hundred Kelvin. In particular, $W 1-W 2$ colours of 3, 2, and 1 mags correspond to black body temperatures of 350,500 , and $900 \mathrm{~K}$, respectively. This colour excess also depends on the relative contribution of hot dust emission to the total emission. Therefore, while it clearly points at hot dust in extreme cases, the $W 1-W 2$ colour alone is not sufficient for a precise determination of the dust temperature. The spectral energy distribution is needed in a wide wavelength range to fit and subtract stellar and gaseous emission.

In Fig. 1a we show the dependence of $W 1-W 2$ on the oxygen abundance $12+\log \mathrm{O} / \mathrm{H}$ for the sample of $\sim 1000$ galaxies with the best determined oxygen abundances (see Sect. 2). The sample is split into two subsamples of objects with the $\mathrm{H} \beta$ luminosity $L(\mathrm{H} \beta) \geq 3 \times 10^{40} \mathrm{erg} \mathrm{s}^{-1}$ (small red filled circles) corresponding to a star-formation rate SFR $\geq 0.7 M_{\odot} \mathrm{yr}^{-1}$ (according to prescriptions of Kennicutt 1998) and $L(\mathrm{H} \beta)<$ $3 \times 10^{40} \mathrm{erg} \mathrm{s}^{-1}$ (small blue open circles). Figure $1 \mathrm{~b}$ shows the histogram of colour distributions for both samples. It is seen from Figs. 1a,b that a major fraction of galaxies has 
Y. I. Izotov et al.: New star-forming galaxies with hot dust emission
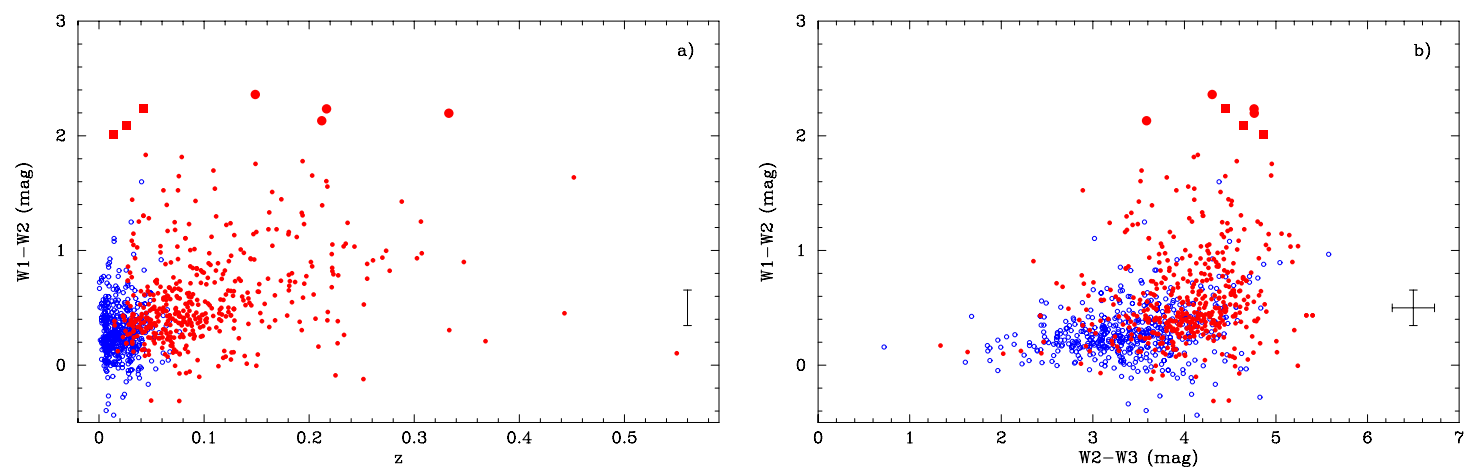

Fig. 2. a) Dependence of $W 1-W 2$ colour on redshift $z$ for the sample of $\sim 1000$ galaxies. b) $(W 1-W 2)$ vs. (W2 - W3) colour-colour diagram. Symbols are the same as in Fig. 1a.

Table 1. Properties of newly identified star-forming galaxies with red $W 1-W 2$ colours.

\begin{tabular}{|c|c|c|c|c|}
\hline Property & $\overline{\mathrm{J} 0219+0018}$ & $\bar{J} 1439+2453$ & $\bar{J} 1457+2232$ & $\mathrm{~J} 1514+3852$ \\
\hline$\overline{\mathrm{RA}(\mathrm{J} 2000)}$ & 02:39:00.79 & $14: 39: 05.24$ & $14: 57: 35.14$ & $15: 14: 08.63$ \\
\hline $\operatorname{Dec}(J 2000)$ & $+00: 18: 35.88$ & $+24: 53: 53.39$ & $+22: 32: 01.79$ & $+38: 52: 07.31$ \\
\hline$z$ & 0.2166 & 0.2119 & 0.1488 & 0.3329 \\
\hline SDSS $g$ (mag) & 20.72 & 19.54 & 19.43 & 20.44 \\
\hline$W 1$ (mag) & $17.80 \pm 0.28$ & $14.26 \pm 0.03$ & $16.22 \pm 0.08$ & $17.60 \pm 0.23$ \\
\hline$W 2$ (mag) & $15.57 \pm 0.16$ & $12.13 \pm 0.02$ & $13.85 \pm 0.05$ & $15.40 \pm 0.12$ \\
\hline W3 (mag) & $10.81 \pm 0.09$ & $8.55 \pm 0.03$ & $9.55 \pm 0.04$ & $10.64 \pm 0.07$ \\
\hline W4 (mag) & $7.65 \pm 0.12$ & $6.16 \pm 0.05$ & $6.54 \pm 0.06$ & $7.77 \pm 0.13$ \\
\hline$L(\mathrm{H} \beta)\left(\mathrm{erg} \mathrm{s}^{-1}\right)$ & $2.8 \times 10^{41}$ & $1.2 \times 10^{42}$ & $4.8 \times 10^{41}$ & $2.6 \times 10^{41}$ \\
\hline $\operatorname{SFR}(\mathrm{H} \beta)\left(M_{\odot} \mathrm{yr}^{-1}\right)$ & 6.1 & 26.5 & 10.6 & 5.8 \\
\hline$M_{*} / M_{\odot}{ }^{a}$ & $1.9 \times 10^{8}$ & $2.9 \times 10^{8}$ & $1.3 \times 10^{9}$ & $2.1 \times 10^{9}$ \\
\hline$M$ (burst) $/ M_{\odot}{ }^{a}$ & $3.0 \times 10^{7}$ & $9.9 \times 10^{7}$ & $4.7 \times 10^{7}$ & $4.8 \times 10^{7}$ \\
\hline $\mathrm{EW}(\mathrm{H} \beta)(\AA)$ & 155 & 174 & 205 & 105 \\
\hline$t$ (burst) $(\mathrm{Myr})^{a}$ & 3.9 & 3.6 & 3.1 & 3.9 \\
\hline $12+\log \mathrm{O} / \mathrm{H}$ & 8.08 & 8.09 & 8.04 & 8.40 \\
\hline
\end{tabular}

Notes. ${ }^{(a)}$ Stellar masses $M_{*}$, starburst masses $M$ (burst) and ages $t$ (burst) are derived from SDSS spectra according to prescriptions of Izotov et al. (2011).
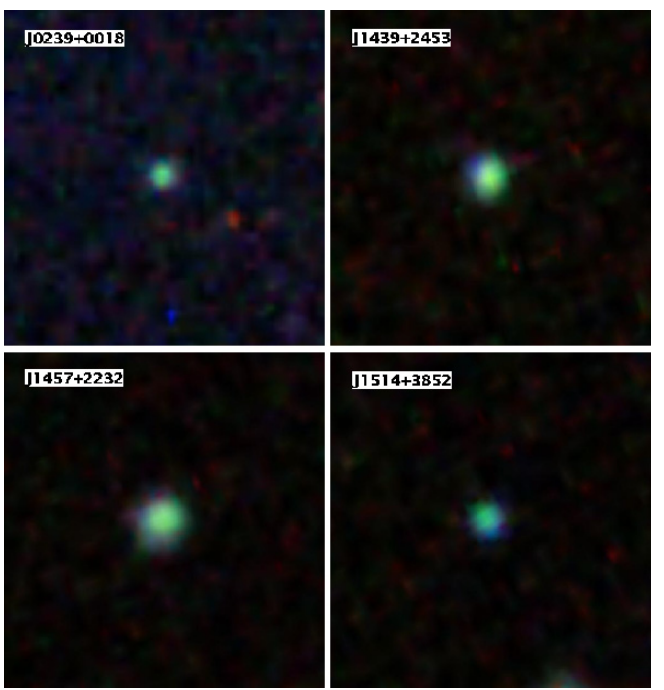

Fig. 3. $20^{\prime \prime} \times 20^{\prime \prime}$ SDSS images of galaxies with $W 1-W 2>2$ mag.

$W 1-W 2 \leq 0.5 \mathrm{mag}$, implying that emission in these galaxies is dominated by stars and ionised gas. On the other hand, there are 65 galaxies with $W 1-W 2 \sim 1-2$ mag where the contribution of hot dust emission is significant. Most of these galaxies are characterised by high SFRs $\geq 0.7 M_{\odot} \mathrm{yr}^{-1}$ and can therefore be

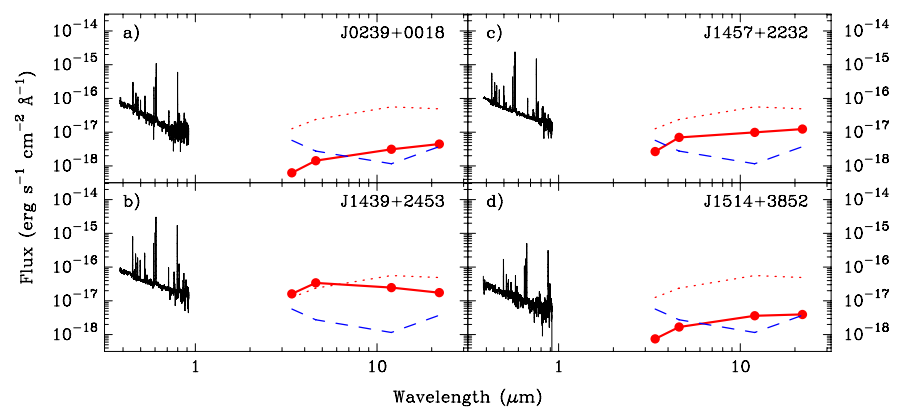

Fig. 4. Observed spectral energy distributions (SED) of galaxies with $W 1-W 2>2$ mag, which include SDSS optical spectra (black solid lines) and WISE MIR photometric data in all four bands (red symbols and solid lines). For comparison in all panels are shown MIR SEDs for the "active" BCD SBS 0335-052E (red dotted lines) and for the "passive" BCD I Zw 18 (blue dashed lines).

classified as LCGs (Izotov et al. 2011) or "active" galaxies following the nomenclature of Hirashita \& Hunt (2004). No colour dependence on metallicity is present, at variance with the conclusion made by Griffith et al. (2011) on the base of a smaller BCD sample.

In Fig. 1c,d the same sample is split into sources with low and high $\mathrm{H} \beta$ equivalent widths $\operatorname{EW}(\mathrm{H} \beta)$, which is a measure of star formation burst age (high values relate to very recent bursts). 
Clearly, red $W 1-W 2$ colours are observed mainly in galaxies with very young $(<3-4 \mathrm{Myr})$ starbursts.

Figures 2a,b present the dependence of $W 1-W 2$ colours on the redshift $z$ and the $(W 1-W 2)-(W 2-W 3)$ colour-colour diagram, respectively, for the same sample as in Fig. 1 split into two parts with $L(\mathrm{H} \beta) \geq 3 \times 10^{40} \mathrm{erg} \mathrm{s}^{-1}$ (red symbols) and $L(\mathrm{H} \beta)<$ $3 \times 10^{40} \mathrm{erg} \mathrm{s}^{-1}$ (blue symbols). No evident trend of the $W 1-W 2$ colour on $z$ is present for galaxies with $W 1-W 2 \geq 1 \mathrm{mag}$, while for galaxies with bluer $W 1-W 2$ colours there is a tendency to be redder with increasing $z$. The colour-colour diagram (Fig. 2b) shows that galaxies with low SFRs (blue symbols) occupy the region of spiral galaxies, according to Lake et al. (2011). On the other hand, galaxies with high SFRs (red symbols) are mixed with QSOs, Seyferts, ultraluminous infrared galaxies (ULIRGs), LINERs and starbursts. Physical properties of our galaxies are very different from those of other types of galaxies, excluding starbursts. Evidently, the colour-colour diagram in Fig. $2 b$ fails to separate galaxies of different types.

Coming back to Figs. 1a,c, it can also be seen that galaxies with very red colours $W 1-W 2>2$ mag are rare. Griffith et al. (2011) found three galaxies (red filled squares) that are not present in our sample, because no SDSS spectra are available for them. We find only four more galaxies like these (large red filled circles) out of $\sim 5000$ galaxies from our SDSS sample with the available WISE data. In the colour-colour diagram our four objects together with three galaxies from Griffith et al. (2011); (large symbols in Fig. 2b) are located in the region of ULIRGs, LINERs and obscured AGNs, but their other properties are very different. General characteristics of the newly identified four galaxies are shown in Table 1, SDSS images and observed spectral energy distributions (SEDs) are shown in Figs. 3 and 4. All four galaxies are very compact $\left(\sim 1-2^{\prime \prime}\right.$ in diameter, corresponding to linear scales $\sim 3-6 \mathrm{kpc}$ at their redshifts of $0.15-0.33$ ) and are almost unresolved. The SDSS spectra resemble those with strong emission lines, which is suggestive of active starformation in young bursts with rates SFR 6-27 $M_{\odot} \mathrm{yr}^{-1}$. Their MIR SEDs (red symbols and solid lines in Fig. 4) indicate a flux excess similar to that in the "active" BCD SBS 0335-052E (red dotted lines) in contrast to no flux excess in the "passive" BCD I Zw 18 (blue dashed lines). Their low stellar masses of $\sim 10^{8}-10^{9} M_{\odot}$ (Table 1) derived from the fitting of SDSS spectra are characteristic of dwarf galaxies according to Izotov et al. (2011).

Certainly, these galaxies can be classified as "green pea" galaxies (Cardamone et al. 2009) or LCGs (Izotov et al. 2011). The characteristics of the newly identified four galaxies with very red $W 1-W 2$ colours are similar to those in high-redshift LBGs. On the other hand, oxygen abundances in these galaxies are higher than in the three BCDs discussed by Griffith et al. (2011), indicating that there is no apparent dependence on metallicity. In the sample of 803 LCGs by Izotov et al. (2011) there are many other galaxies with optical characteristics similar to the LCGs in Table 1 but with more moderate characteristics in the MIR range. Probably, high $\mathrm{H} \beta$ luminosity or young age of the burst is not the only factor for heating the dust to high temperatures. Other factors may play a role, such as the morphology and compactness of the galaxy, the presence of super-star clusters (SSCs), distribution of the interstellar gas and dust in the vicinity of young clusters. Therefore, Hubble Space Telescope (HST) high-angular resolution imaging, spectroscopic observations in a wide wavelength range including the MIR range and interferometric observations in the $\mathrm{H}_{\mathrm{I}} \lambda 21 \mathrm{~cm}$ line and in the radio-continuum will be useful for a better understanding of the origin of hot dust and the determination of its properties.

\section{Summary}

We carried out a search for star-forming Sloan Digital Sky Survey (SDSS) galaxies with strong emission lines in the Widefield Infrared Survey Explorer (WISE) Preliminary Release Source Catalogue (PRSC) aiming to find galaxies with hot dust emission at wavelengths $\lambda 3.4-4.6 \mu \mathrm{m}$ ( $W 1$ and $W 2$ bands). We find that $\sim 5000$ galaxies out of the total sample of $\sim 16000$ SDSS galaxies are present in the PRSC, which covers only $\sim 57 \%$ of the sky. About 1000 galaxies out of the galaxies detected with WISE have a sufficiently strong [O III] $\lambda 4363$ emission line in their SDSS spectra to allow a reliable abundance determination. The comparison of optical and mid-infrared properties for the sample of galaxies with reliably derived oxygen abundances led us to following conclusions:

A major fraction of galaxies has $W 1-W 2$ of $\sim 0.0-0.4$ mag, consistent with the colour for the emission from stars and the ionised interstellar medium. The contribution of hot dust emission is small. On the other hand, there are 65 galaxies with redder $W 1-W 2$ colours, $\sim 1-2$ mag. Most of these galaxies are luminous compact galaxies (LCGs) with star-formation rates SFR $>0.7 M_{\odot} \mathrm{yr}^{-1}$ and high $\mathrm{H} \beta$ equivalent widths $E W(\mathrm{H} \beta)>$ $50 \AA$, implying a very recent starburst, that can efficiently heat interstellar dust to high temperatures.

Star-forming galaxies with very red $W 1-W 2$ colours $>2$ mag are rare. In addition to three galaxies previously studied by Griffith et al. (2011), which are not present in our sample because of the lack of SDSS spectra for them, we find only four galaxies like these from the sample of $\sim 5000$ galaxies detected with WISE.

Acknowledgements. We thank the referee S. Bianchi for valuable comments. Y.I.I., N.G.G. and K.J.F. are grateful to the staff of the Max Planck Institute for Radioastronomy (MPIfR) for their warm hospitality. Y.I.I. and N.G.G. acknowledge a financial support of the MPIfR. This publication makes use of data products from the Wide-field Infrared Survey Explorer, which is a joint project of the University of California, Los Angeles, and the Jet Propulsion Laboratory, California Institute of Technology, funded by the National Aeronautics and Space Administration. Funding for the Sloan Digital Sky Survey (SDSS) and SDSS-II has been provided by the Alfred P. Sloan Foundation, the Participating Institutions, the National Science Foundation, the U.S. Department of Energy, the National Aeronautics and Space Administration, the Japanese Monbukagakusho, and the Max Planck Society, and the Higher Education Funding Council for England.

\section{References}

Abazajian, K. N., Adelman-McCarthy, J. K., Agüeros, M. A., et al. 2009, ApJS, 182, 543

Cardamone, C., Schawinski, K., Sarzi, M., et al. 2009, MNRAS, 399, 1199 Engelbracht, C. W., Rieke, G. H., Gordon, K. D., et al. 2008, ApJ, 678, 804 Griffith, R. L., Tsai, C.-W., Stern, D., et al. 2011, ApJ, 736, L22

Guseva, N. G., Papaderos, P., Meyer, H. T., Izotov, Y. I., \& Fricke, K. J. 2009, A\&A, 505, 63

Heckman, T. M., Hoopes, C. G., Seibert, M., et al. 2005, ApJ, 619, L35

Hirashita, H., \& Hunt, L. K. 2004, A\&A, 421, 555

Houck, J. R., Charmandaris, V., Brandl, B. R., et al. 2004, ApJS, 154, 211

Hoyos, C., Koo, D. C., Phillips, A. C., Willmer, C. N. A., \& Guhathakurta, P. 2005, ApJ, 635, L21

Hunt, L. K., Vanzi, L., \& Thuan, T. X. 2001, A\&A, 377, 66

Hunt, L. K., Thuan, T. X., Izotov, Y. I., \& Sauvage, M. 2010, ApJ, 712, 164

Izotov, Y. I., Guseva, N. G., Lipovetsky, V. A., Kniazev, A. Y., \& Stepanian, J. A. 1990, Nature, 343, 238

Izotov, Y. I., Guseva, N. G., \& Thuan, T. X. 2011, ApJ, 728, 161

Kakazu, Y., Cowie, L. L., \& Hu, E. M. 2007, ApJ, 668, 853

Kennicutt, R. C., Jr. 1998, ARA\&A, 36, 189

Lake, S. E., Wright, E. L., Petty, S., et al. 2011, AJ, 143, 7

Thuan, T. X., Sauvage, M., \& Madden, S. 1999, ApJ, 516, 783

Wright, E. L., Eisenhardt, P. R. M., Mainzer, A. K., et al. 2010, AJ, 140, 1868 